\title{
STUDY ON INSPECTION OF CIRCULAR PARTS USED IN MECHATRONICS
}

\author{
Robert Ciobanu ${ }^{1}$, Dana Rizescu², Ciprian Rizescu ${ }^{3}$ \\ 1,2,3 University Politehnica of Bucharest \\ Splaiul Independenţei, no. 313, Bucharest, Romania \\ robert.ciobanu@upb.ro
}

\begin{abstract}
Classical methods for the inspection on a production line of circular profiles are characterized by a reduced flexibility, making them extremely expensive. This work provides a study on vision systems used for determining deviation from circularity of components used in mechatronics. The paper present a vision system that analyzes and processes the captured image of circular parts in an automatic mode in order to undertake the dimensional inspection. The proposed system has been tested for MR522ZEZO class miniature bearings. The inner diameter of MR522ZEZO bearing is $2 \mathrm{~mm}$, which makes it unavailable for dimensional control by conventional methods. Experimental results show that the proposed system is able to perform an accurate dimensional control in automatic mode while ensuring high flexibility for distinct types and sizes of mechatronic components with circular profiles.
\end{abstract}

Keywords: Optical measurements, thresholding, image denoising, ROI extraction.

\section{Introduction}

Conventional quality control has a decreasing brand share within industry, being replaced by automated vision systems. The metrology of cylindrical parts has a wide range of applicability because in the industry a large number of parts obtained on CNC machines require a series of technical execution conditions that mainly refer to obtaining a more accurate geometric form [1]. Shape deviations of cylindrical parts have a negative influence on the functionality and/or durability of the assemblies they make as adjustments.

The deviation from circularity is an important criterion for accepting or rejecting circular shape parts. Additionally, the measurement results of circular deviations are used for machine tool setting. Shape deviation of revolutionary pieces is measured with respect to the conventionally defined reference circle/circles in a section perpendicular to the axis. The result of measuring circularity deviation is influenced by the following factors: the number of measured points, the distribution of measured points, the evaluation method used. Based on the type of the reference element, four methods have been developed for assessing the deviation from circularity: least squares method, minimum circumscribed circle method, maximum scored circle method, minimal zone method. The most common method used for the analysis of circular profile is the least squares method, which consists in determining a reference circle by minimizing the sum of squared distances from the points on the radial periphery of the circle measured profile.

Mechatronic component processing technologies have seen a quantum leap particular by means of numerical control centers, and the variety of shapes and sizes of components raises a series of problems for the total control of the production flow. These aspects have generated the need of introducing the visual inspection on the production line. Visual inspection in a production line requires dynamic capture of the image and static image processing is an offset in obtaining the mathematical model [2].

Taking into account this consideration, there comes the need to develop a multiobjective optimization that follows the sizing of controlled components within the error limits in a speed condition imposed by the manufacturing flow.

As regards the precision of measuring equipment for circular components obtained on numerical control machine tools, this must be correlated with the dimensional tollerances and shape deviations resulting from technological operations.

The quality and precision class of a visual inspection system is set by the image forming, processing and analysing system $[3,4]$.

Under these conditions, the acquired image must be processed - optimized. Criteria for assessing an image quality are application specific, setting the interactive feature of its optimization quality process and the need for user validation. In conclusion, any original image can be improved by getting a 
preferred image for the type of application in which the visual inspection system is integrated.

For low contrast components and complex shapes, vision systems encounter a number of difficulties and the best solution has not yet been implemented. From a scientific point of view, there are a number of solutions that offer a certain type of lighting or multi-camera systems [5].

\section{Vision System}

The current trend of mechatronic systems and subsystems used in industry as consumer goods is to reduce their sizes while increasing their performance. Miniaturization of the mechatronic components produces changes in the manufacturing flow. One of the work objectives was to develop a visual inspection system and to optimize the process for measuring the diameters of small cylindrical components automatically and in a standardized manner. System development took into account the need for flexibility and adaptability for a wide range of sizes. The results obtained are satisfactory in terms of precision.

The vision system consists of two main parts, namely the hardware part and the software part (fig. $1)$. The hardware elements are used to form and capture the image of the component under dimensional control, and the software component is used to sizing and performing the inspection process by analyzing, processing and sizing the captured image.

Considering that the system addresses a small 0-5 mm component class, a constructive solution has been adopted with an optical image zoom system consisting of:

-microscope: prism body and adjacent tubes;

-microscope objective;

-CMOS video camera, USB 3.0, global shutter, 60 fps;

-image acquisition and processing system.

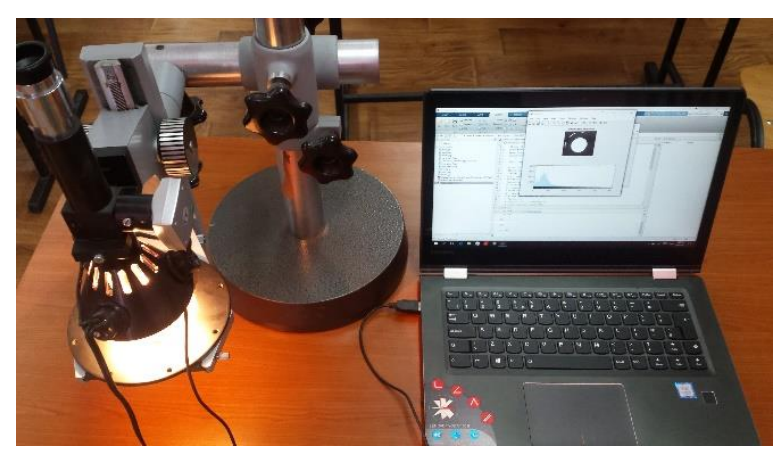

Fig. 1. - Vision system

The studied component is represented by a MR 522Z-EZO bearing with the following catalog data $\mathrm{d}=2$ $\mathrm{mm}, \mathrm{D}=5 \mathrm{~mm}, \mathrm{~B}=2.5 \mathrm{~mm}$. Bearings are parts from the category of components that needs to be subject to a strictly dimensional control.
In this paper, the dimensional control of the inner ring of the bearing was made in static mode with the development perspective of automating the process in a manufacturing line.

The simulations were done both in Matlab and LabView. Because Matlab offers increased flexibility the final algorithm has been developed in Matlab (Fig. 2).
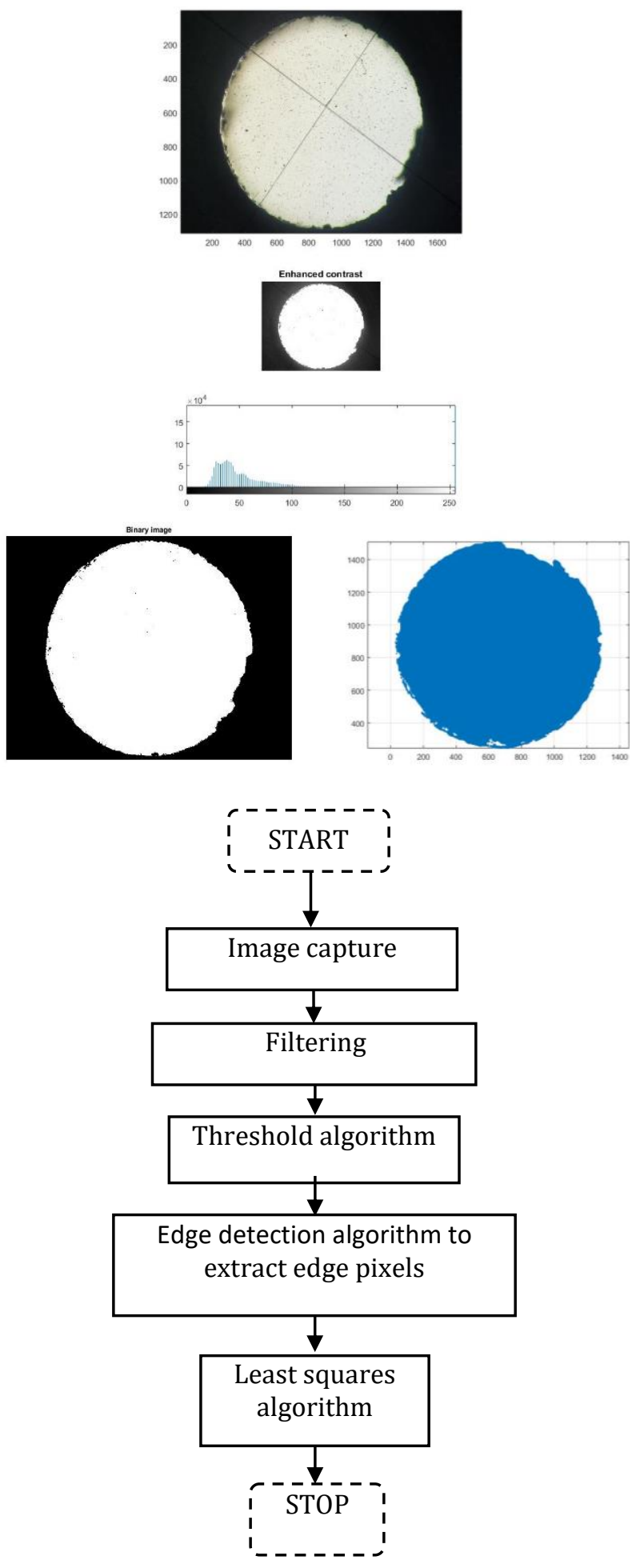

Fig. 2. Algorithm processing steps 


\section{Size Measurement Algorithm}

\subsection{Filtering and edge detection}

Using the Fourier transform, the digital signal is transformed into the frequency range for which the amplitude and signal phase can be determined [6].

Continuous Fourier transform $F(\omega)$ and inverse Fourier transform $\mathrm{f}(\mathrm{t})$ :

$$
\begin{aligned}
& F(\omega)=\int_{-\infty}^{\infty} f(t) e^{-i \omega t} d t \\
& f(t)=\frac{1}{2 \pi} \int_{-\infty}^{\infty} F(\omega) e^{i \omega t} d t
\end{aligned}
$$

This transformation is important because, by suppressing the signals with specific wavelengths, one can better analyze the shape deviations of a particular type. By separating the signals with different wavelengths we can analyze the phenomena that they produce. Fast Fourier Transform (FFT) was applied to the filtering.
This is a more efficient algorithm than the one based on Direct Fourier Transform (DFT) and can also be used to determine the Fourier Inverse Transform (IFT) [6]. The most widely used method of digital signal processing is based on Discrete Fourier Transforms(DFT) that allows the transformation of the time domain signal into the frequency domain, this process being known as spectral analysis. This transformation is important because, by suppressing signals with certain wavelengths, we can better analyze deviations of a certain type. For filtering was applied Fast Fourier Transform (FFT) [6].

$$
\begin{aligned}
& X_{k}(n)=\sum_{n=0}^{N-1} x_{n} e^{-i 2 \pi k n / N}, \\
& \mathrm{k}=0,1 \ldots, \mathrm{N}-1
\end{aligned}
$$

The Fast Fourier Transform algorithm used is more efficient than the one based on Discrete Fourier Transform (DFT) and can also be used to determine the Inverse Fourier Transform (IFT).

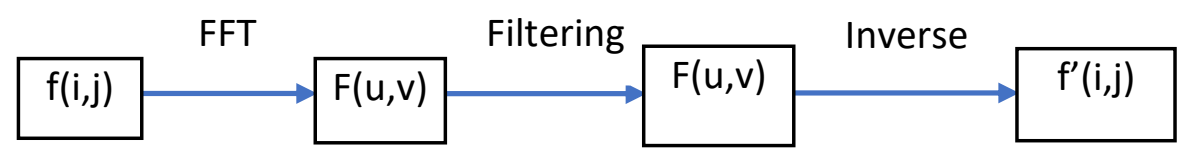

Edge detection is used to find locations in the digital image where the image brightness changes abruptly along a line of pixels [7]. These abrupt changes usually define the edge of an object in an image. By filtering the image the contours of inner ring is defined more clearly. Using the edge detection, boundaries of the inner ring can be identified. Once the boundary of the inner ring is located, the size as well as other features can be determined.

In terms of edge detection a hybrid algorithm was developed in Matlab based on Canny [8] and fuzzy logic methods.

\subsection{Least squares fitting of circles}

For the set of pixels $\mathrm{N}$ that describe the circular profile of a part is considered $0(0,0)$ the origin of the system and $\mathrm{O}_{\mathrm{LS}}(\mathrm{a}, \mathrm{b})$ the origin of the workpiece coordinate system. $\mathrm{P}$ is a pixel on the traced profile, whose position in the equipment coordinate system is given by the angle $\theta_{i}$ and the distance $R_{i}$.

The deviation of the profile at point $\mathrm{P}$ unto the circle of the least squares is $\Delta$ (fig. 3 ).

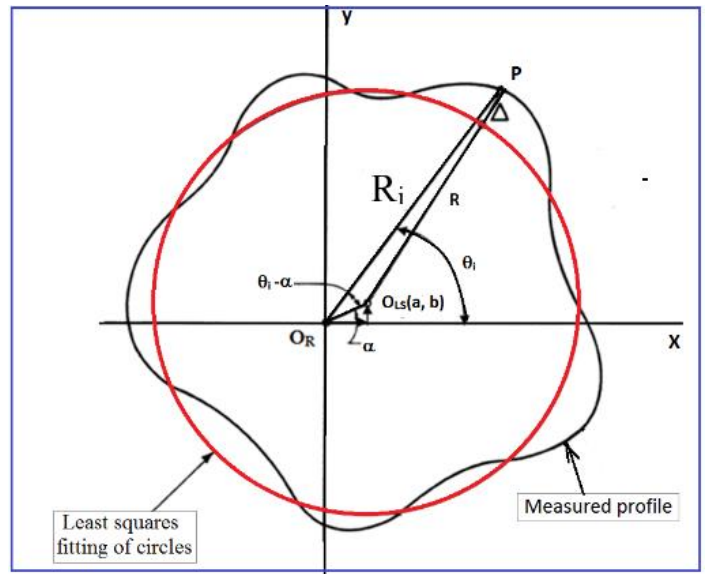

Fig. 3. Deviation from circularity

Determining on the circumference of measured profile " $n$ " points $\mathrm{P}_{\mathrm{i}}$, equidistant (angular), defined by the angles $q_{i}(i=1 \ldots n)$, the sum of square profile deviations in Pi points relative to least squares circle $\left(\mathrm{S}=\sum_{i=1}^{n} \Delta_{i}^{2}\right)$ can be minimized by the least squares fitting of circles[9,10]. Thus by deriving equation $\mathrm{S}$ in function of $\mathrm{a}, \mathrm{b}$ and $\mathrm{R}$ and equaling zero the following relationships are obtained: 


$$
\begin{aligned}
& a=\frac{2}{n} \sum_{i=1}^{n} R_{i} \cos \theta_{i} ; b=\frac{2}{n} \sum_{i=1}^{n} R_{i} \sin \theta_{i} ; \\
& \mathrm{R}=\frac{1}{\mathrm{n}} \sum_{\mathrm{i}=1}^{\mathrm{n}} \mathrm{R}_{\mathrm{i}}
\end{aligned}
$$

\section{Experimental Results}

The tests were performed in static mode, defining an optimal sizing algorithm. Higher quality images are important for reducing processing time and therefore optimizing the inspection process. Considering also the low contrast level of the image, two lighting methods were tested, namely front lighting and backlighting (fig. 4).

In case of backlight, the high contrast between the area of interest and the other areas makes it possible to detect edges smoothly without running specific filters from the processing algorithm. In terms of processing time by removing those steps the time is reduce with $30 \%$. This aspect is extremely important on an automated process.

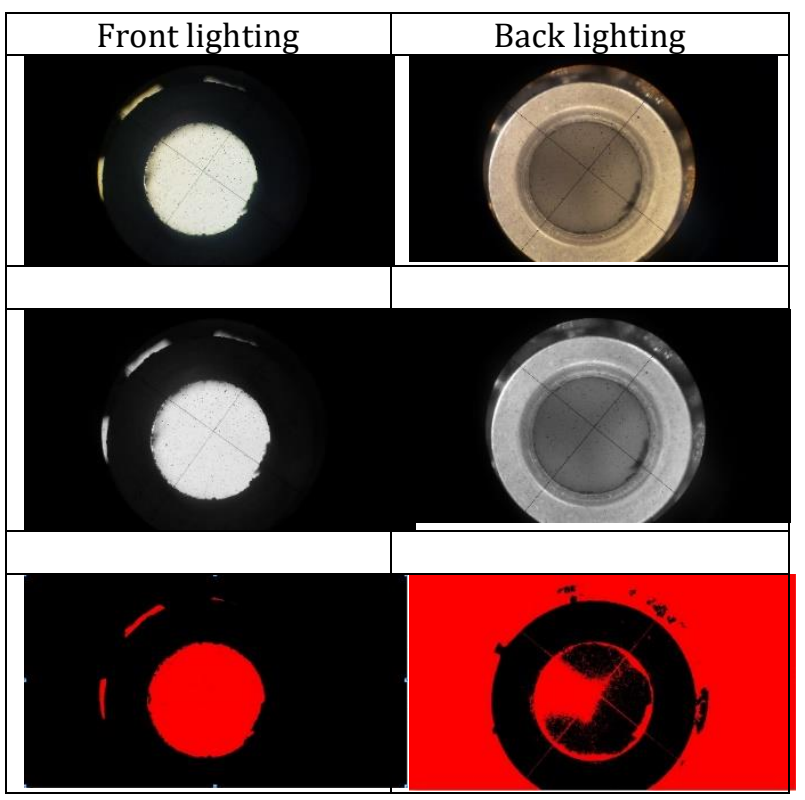

Fig. 4. Front and back lighting

Having as an objective to obtain a reduced time for processing the image, edge detection and establish the dimension of the inner ring of bearing, both LabVIEW and MatLab were tested. LabView is more efficient and easy to implement compared to MATLAB in real time application. As an negative aspect, LabView has limitations regarding atypical situations.

The algorithm proposed was developed in Matlab. After filtering and denoising the picture, edge detection was performed and the profile of the inner ring was obtained (fig. 5). The profile was optimized using least squares fitting of circles algorithm.

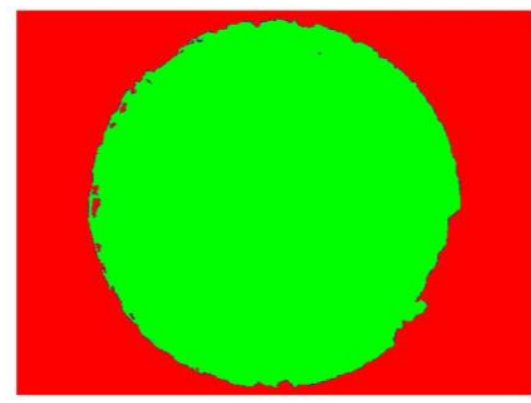

a)

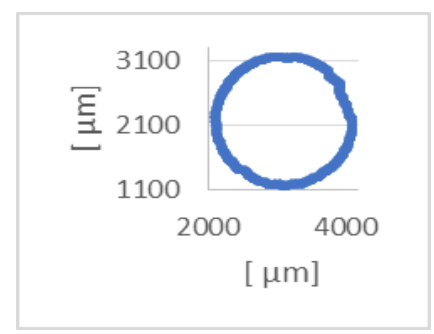

b)

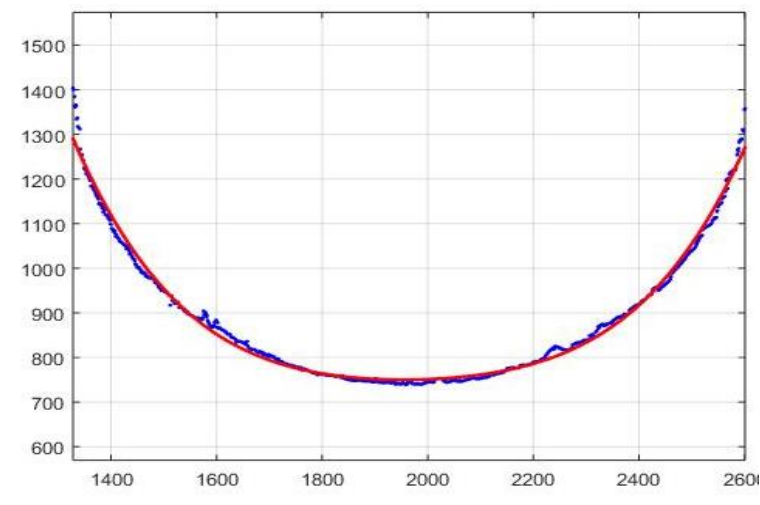

c)

Fig. 5. Matlab results: a) filtered and denoised picture, b) edge detection, c) least squares fitting of circles

In conditions of a mid-range image acquisition system the part studied has been dimensioned with an increased level of precision at a low execution time of about 1.5 seconds. The measurement accuracy is about 10 microns.

\section{Conclusion}

The analysis of the results above shows that the proposed system has a better measuring accuracy than the standards for class of bearings subject to inspection.

The image processing algorithm developed returns good results.

In the situation of inspecting parts in a low contrast environment the algorithm must be updated, meaning that the inspection process time will increase. 
Two of the most important characteristic of an vision system are the precision and the time of evaluating the product.

Modern manufacturing technologies have allowed the miniaturization of elements requiring high precision. Under these conditions, the development of vision systems with optical amplification subsystems needs to be developed.

The theoretical foundaments of the harmonic analysis of circular profiles helps to determine the conditions of analysis of the inspected profiles and the choice of filters and to identify the causes that generate different shape deviations during the manufacturing process.

Any original image can be improved by getting a preferred image for the type of application in which the visual inspection system is integrated.

\section{Acknowledgements}

This work has been funded by University Politehnica of Bucharest, through the "Excellence Research Grants" Program, UPB - GEX. Identifier: UPBEXCELENȚĂ-2016, Vision system for active dimensional control of mechatronic components in a production line, contract number 41/26.09.2016.

\section{References}

[1] Golden, C.L.: Analysis of Form Errors in Rings of Non-Uniform Cross Section due to Workholding and Machining Loads, Paper for the Degree Master of Science in Mechanical Engineering, George W. Woodruf School of Mechanical Engineering Georgia Institute of Technology, May (2008).
[2] Malamas, E.N., Petrakis, E.G.M., Zervakis, M. Petit, L., Legat, J.D.: A survey on industrial vision systems, applications, and tools, Image Vis. Comput. 21, 171-188, (2003).

[3] Mak, K.L. Peng, P.: An automated inspection system for textile fabrics based on Gabor filters, Robot. Comput. Int. Manuf. Arch. 24 (3), 359369, (2008).

[4] Ngan, Henry Y.Y., Pang, Grantham K.H., Yung, Nelson H.C.: Automated fabric defect detection a review, Image Vis. Comput. 29, 442-458, (2011).

[5] Psarakis, E.Z., Evangelidis, G.D.: An enhanced correlation-based method for stereo correspondence with subpixel accuracy, IEEE International Conference on Computer Vision, 2005, ICCV2005,vol.1, pp.907-912, (2005).

[6] Gonzalez, Rafael C., Woods, Richard E., and Eddins, Steven, L.: Digital Image Processing, Using MATLAB;

[7] Shijie, Feng, QianChen a, ChaoZuo a,b, Asundi Anand: Fast three-dimensional measurements for dynamic scenes with shiny surfaces, OpticsCommunications382, 18-27, (2017).

[8] Canny, J.: A computational approach to edge detection. IEEE Transactions on Pattern Analysis and Machine Intelligence 8 (6), 679-698, (1986).

[9] Shen, H. , Shuxiao Li, Duoyu Gu, Chang H.: Bearing defect inspection based on machine vision, Measurement 45, 719-733, (2012).

[10] Chernov, N. and Lesort, C.: Least squares fitting of circles and lines, February 1, Department of Mathematics University of Alabama at Birmingham Birmingham, AL 35294, USA; (2008). 